\title{
SÍNDROME DE WEST: REPORTE DE UN CASO
}

\section{WEST SYNDROME: CASE REPORT}

\section{Carlos Miguel RIOS-GONZÁLEZ ${ }^{1}$ Jessica FLOREZ-ENRIQUEZ².}

${ }^{1}$ Facultad de Ciencias Médicas, Universidad Nacional de Caaguazú, Coronel Oviedo - Paraguay.

${ }^{2}$ Facultad de Ciencias Médicas, Universidad Central de Ecuador, Quito - Ecuador.

Cómo citar este artículo: Rios-González C, Florez-Enriquez J. Síndrme de West: reporte de un caso. Medicina Clínica y Social. 2018;2(1):37-40.

\section{RESUMEN}

Es una encefalopatía epiléptica dependiente de la edad caracterizada por la tríada electroclínica de espasmos epilépticos, retardo del desarrollo psicomotor y patrón electroencefalográfico de hipsarritmia en el electroencefalograma. El objetivo fue reportar un caso de Síndrome de West (SW) en paciente ecuatoriano. Paciente de sexo masculino de 6 meses de edad, consulta por manifestar 3 crisis convulsivas en menos de 24 horas con breve pérdida de la conciencia de aproximadamente 3 minutos y revulsión ocular, acompañadas de espasmos que se caracterizan por la contracción bilateral, brusca y simétrica de músculos cervicales, tronco y miembros. Se evidencia alteración en el desarrollo motor al no concordar con su edad cronológica. En el EEG se evidencia un patrón de hipsarritmia con lo cual se llega a un consenso diagnóstico del síndrome de West con espasmos en extensión, hipotonía e hipoactividad, asimismo en el EEG se evidenció frecuentes descargas epileptiformes focales con ondas lentas y complejos de puntas. Es importante para profesionales en salud considerar como diagnóstico de SW en pacientes que presenten el cuadro clínico mencionado, para alcanzar un mejor pronóstico, ya que al no ser manejado de manera adecuada y oportuna tendrá graves repercusiones en el paciente

Palabras clave: Espasmos Infantiles; Convulsiones; Síndrome de West.

\section{ABSTRACT}

It is an age-dependent epileptic encephalopathy characterized by the electroclinical triad of epileptic spasms, delayed psychomotor development and electroencephalographic pattern of hypsarrhythmia in the electroencephalogram. The goal was to report a case of West syndrome in an Ecuadorian patient. A 6-month-old male patient was consulted for 3 seizures in less than 24 hours with brief loss of consciousness of approximately 3 minutes and ocular revulsion, accompanied by spasms characterized by bilateral, abrupt contraction and symmetrical cervical muscles, trunk and limbs. Alteration in motor development is evidenced by not agreeing with its chronological age. In the EEG, a pattern of hypsarrhythmia is evident, which leads to a diagnostic consensus of the West syndrome with spasms in extension, hypotonia and hypoactivity. Likewise, EEG showed frequent focal epileptiform discharges with slow and complex waves of points. It is important for health professionals to consider SW diagnosis in patients presenting the mentioned clinical picture, in order to reach a better prognosis, since not being handled in an adequate and timely manner will have serious repercussions on the patient

Keywords: Infantile spasms; Seizures; West syndrome. 


\section{INTRODUCCIÓN}

El síndrome de West (SW) es un síndrome encefalopático epiléptico, se presenta principalmente en niños menores de dos años de edad (1), definido por una tríada de espasmos infantiles, patrón de disritmia lenta con puntas-ondas difusas en el electroencefalograma (EEG) y paro o regresión de desarrollo (2).

Se estima que la frecuencia oscila entre $2 \%$ y $10 \%$, con una incidencia de 1 cada 4000 a 6000 nacidos vivos $(3,4)$. Se estima que el $80 \%$ de las personas afectadas viven en países en vías desarrollo (1). Sin un tratamiento adecuado, la crisis afecta al desarrollo normal de los bebes, demostrando signos de regresión de su desarrollo psicomotor (4). Los casos adquiridos incluyen secuelas de infecciones del Sistema Nervioso Central (SNC), lesiones isquémicas hipóxicas y postraumáticas, mientras que los SW congénitos son genéticos, resultando principalmente de mutaciones en genes únicos que comparten vínculos funcionales. Hasta ahora las mutaciones en ARX, CDKL5, STXBP1, SLC25A22 y SPTAN1 ya han sido vinculadas al SW (5).

Se clasifica de acuerdo con la etiología subyacente en 1- SW adquirido, 2- SW congénito/de desarrollo, y 3- SW de etiología desconocida (6). El pronóstico es generalmente grave, 7090\% de los pacientes desarrollan retrasos mentales y $20-50 \%$ el síndrome de Lennox-Gastaut (7). El diagnóstico se basa principalmente en reconocer la triada: Espasmo infantiles, alteración del desarrollo psicomotor y EEG hipsarrítmico (8).

El tratamiento debe iniciarse dentro de las dos primeras semanas después de iniciados los espasmos. Como fármaco de elección es el derivado sintético de hormona adrenocorticotropa (ACTH) y la vigabatrina mostrando una efectividad de $65 \%$ aproximadamente para el control de crisis, como fármaco de segunda línea se encuentra la vigabatrina la cual presenta limitaciones por sus efectos adversos a nivel visual, una tercera opción de tratamiento son algunos corticoides $(9,10)$. El objetivo de la presente es reportar un caso de síndrome de West de un paciente ecuatoriano.

\section{PRESENTACIÓN DEL CASO CLÍNICO}

Paciente de sexo masculino de 6 meses de edad, nacido y residente en la ciudad de Quito Ecuador, ingresó al servicio de urgencias del Hospital Pediátrico Baca Ortiz por manifestar 3 crisis convulsivas en menos de 24 horas con breve pérdida de la conciencia de aproximadamente 3 minutos y revulsión ocular, acompañadas de espasmos que se caracterizan por la contracción bilateral, brusca y simétrica de músculos cervicales, tronco y miembros.

No refiere antecedentes familiares de interés, sus antecedentes personales son: embarazo de curso normal, parto vaginal a término y sin complicaciones, APGAR 8, inmunización completa de acuerdo al esquema de vacunación del Ministerio de Salud Pública de Ecuador, seno materno exclusivo hasta los 6 meses, 15 días antes del ingreso inicia alimentación complementaria con frutas (manzana, plátano, granadilla, sandia).

Al realizar examen físicos los médicos decidieron mantenerlo en seguimiento y remitirlo al servicio de neuropediatría, en donde se destaca hipotonía e hipoactividad del paciente por lo que se le solicita realizar un electroencefalograma (EEG) por presunción diagnóstica de 
síndrome de West, el cual evidencia un patrón de hipsarritmia con lo cual se llega a un consenso diagnóstico del síndrome de West con espasmos en extensión, hipotonía e hipoactividad, asimismo en el EEG se evidenció frecuentes descargas epileptiformes focales con ondas lentas y complejos de puntas.

A demás se realizan exámenes complementarios como: Tomografía Axial Computarizada (TAC) cerebral demostrándose signos inespecíficos de atrofia cerebral cortical, en la Imagen de Resonancia Magnética (IRM) se apreciaron imágenes de atrofia cerebral inespecífica que no difieren de lo observado en la TAC. Se decide iniciar tratamiento con hidrocortisona $(15 \mathrm{mg} / \mathrm{kg})$ con lo que dos semanas después muestra una excelente evolución valorada con un nuevo EEG.

\section{DISCUSIÓN}

El Dr. William James West, describió por primera vez la condición en su hijo de 4 meses de edad en 1841 (11). Este síndrome es una constelación de síntomas caracterizados por espasmos epilépticos/infantiles, patrones de ondas cerebrales anormales llamados hipsarritmia e incapacidad intelectual (12). Estos espasmos usualmente comienzan en los primeros meses después del nacimiento, que muchas veces pueden ser ayudados con medicamentos (13), en el caso de este paciente la madre niega antecedentes de interés y consumo de algún fármaco, pero es importante destacar la presencia de alteración en el desarrollo motor al no concordar con su edad cronológica. En la actualidad, la Liga Internacional contra la Epilepsia (ILAE) ha revisado la terminología y los espasmos epilépticos ahora se utilizan preferentemente para abarcar los diferentes grupos de edad de inicio (6).

Nuestro paciente presentó la triada clásica de este síndrome lo cual posibilitó el diagnóstico rápido y el inicio precoz del tratamiento, ya que fue atendido en un hospital de tercer nivel, sin embargo, el diagnóstico puede presentar limitaciones en centros de salud de primer nivel donde no disponen de estudios de EEG ni de especialistas neuropediatras.

Es importante para profesionales en salud considerar como diagnóstico al SW en pacientes que presenten el cuadro clínico mencionado, para alcanzar un mejor pronóstico, ya que al no ser manejado de manera adecuada y oportuna tendrá graves repercusiones en el paciente.

\section{CONFLICTOS DE INTERÉS Y FUENTE DE FINANCIACIÓN}

Los autores declaran no poseer conflictos de interés. Fuente de financiación: ninguna.

\section{REFERENCIAS BIBLIOGRÁFICAS}

1. World Health Organization. Epilepsy in the WHO Africa region, Bridging the Gap: The Global campaign against epilepsy "Out of the Shadows". Brazzaville, Congo: World Health Organization 2004. URL.

2. Kato M. A new paradigm for West syndrome based on molecular and cell biology. Epilepsy Res. 2006;70 Suppl 1:S87-95. https://doi.org/10.1016/i.eplepsyres.2006.02.008 
3. Campos MR, Cabello BM, Garrido MM, Martínez BB, Portal IR del, FernándezMensaque RC, et al. Síndrome de West: etiología, opciones terapéuticas, evolución clínica y factores pronósticos. Revista de neurología. 2011;52(2):81-89. URL.

4. Medina P. Síndrome de West, el desafío de una atención oportuna. Revista de NeuroPsiquiatría. 2015;78(2):63-64. URL.

5. Pavone P, Striano P, Falsaperla R, Pavone L, Ruggieri M. Infantile spasms syndrome, West syndrome and related phenotypes: what we know in 2013. Brain Dev. 2014 Oct;36(9):739-51. https://doi.org/10.1016/i.braindev.2013.10.008

6. Paciorkowski AR, Thio LL, Dobyns WB. Genetic and Biologic Classification of Infantile Spasms. Pediatric Neurology. 2011;45(6):355-367. https://doi.org/10.1016/i.pediatrneurol.2011.08.010

7. Berg AT, Berkovic SF, Brodie MJ, Buchhalter J, Cross JH, van Emde Boas W, et al. Revised terminology and concepts for organization of seizures and epilepsies: report of the ILAE Commission on Classification and Terminology, 2005-2009. Epilepsia. 2010;51(4):676-685. https://doi.org/10.1111/i.1528-1167.2010.02522.x

8. Martinez-Quezada DI. Sindrome West. Rev Med MD. 2010;2(1):10-14. URL.

9. Trevathan E, Murphy CC, Yeargin-Allsopp M. The Descriptive Epidemiology of Infantile Spasms Among Atlanta Children. Epilepsia. 1999;40(6):748-51. https://doi.org/10.1111/i.1528-1157.1999.tb00773.x

10. National Clinical Guideline Centre (UK). The Epilepsies: The Diagnosis and Management of the Epilepsies in Adults and Children in Primary and Secondary Care: Pharmacological Update of Clinical Guideline 20. London: Royal College of Physicians (UK); 2012

11. Djuric M, Kravljanac R, Tadic B, Mrlješ-Popovic N, Appleton RE. Long-term outcome in children with infantile spasms treated with vigabatrin: A cohort of 180 patients. Epilepsia. 2014;55(12):1918-1925. https://doi.org/10.1111/epi.12847

12. Auvin S, Hartman AL, Desnous B, Moreau A-C, Alberti C, Delanoe C, et al. Diagnosis delay in West syndrome: misdiagnosis and consequences. Eur J Pediatr. 2012;171(11):1695-1701. https://doi.org/10.1007/s00431-012-1813-6

13. Mytinger JR, Joshi S. The Current Evaluation and Treatment of Infantile Spasms Among Members of the Child Neurology Society. J Child Neurol. 2012;27(10):12891294. https://doi.org/10.1177/0883073812455692 